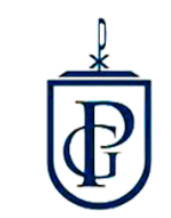

Veritas Et Scientia

Vol. $7, N^{\circ} 1,800-808$

Enero - Junio del 2018.

ISSN $2307-5139$

\title{
DINÁMICA PROYECCIONAL DE LAS MIGRACIONES FRONTERIZAS Y SU INCIDENCIA EN LA RECAUDACIÓN DEL ARANCEL DE ZOFRATACNA, PERIODO 2010-2016
}

PROJECTIONAL DYNAMICS OF BORDER MIGRATIONS AND THEIR INCIDENCE IN THE COLLECTION OF THE TARIFF OF ZOFRATACNA, PERIOD 2010-2016

\section{YESSENIA SOSA JOAQUÍN ${ }^{1}$}

Presentado: $18 / 11 / 2017$

\section{RESUMEN}

El presente estudio de investigación tiene por objetivo principal analizar la estructura de la dinámica de las migraciones fronterizas y su incidencia en la recaudación del arancel de ZOFRATACNA, periodo 2010-2016. Investigación correlacional que pretende demostrar si el comportamiento de la dinámica de las migraciones fronterizas tiene incidencia en la recaudación del arancel de ZOFRATACNA en el periodo 2010-2016 proyectado hacia el año 2019. La evolución de la recaudación trimestral del arancel especial $6 \%$, expresado en miles de dólares, periodo 2010-2016 y su proyección al 2019 tiene una tendencia decreciente. La recaudación del arancel especial del $6 \%$ no tiene relación con la dinámica de las migraciones fronterizas. La ZOFRATACNA no ha planificado en sus documentos de gestión las estrategias, planes, actividades que se orienten a identificar el "negocio para sus clientes -usuarios", tampoco para conocer los gustos y preferencias de los "consumidores con capacidad de compra" que visita la Zona Comercial de Tacna (mercadillos). Existe una alta rotación de los cuadros gerenciales de las unidades orgánicas. Hay una Tasa de deserción del $6 \%$ de los clientes- usuarios actuales, en los últimos 3 años. El ingreso de mercancías desde Chile, con destino a la Zona Comercial de Tacna, representa en promedio el $35 \%$ del total de las importaciones, generando una alta dependencia.

Palabras Clave: cliente, arancel, zona comercial, recaudación.

\footnotetext{
${ }^{1}$ Magíster e Administración y Dirección de Empresas
} 


\begin{abstract}
The main objective of this research study is to analyze the structure of border migration dynamics and its impact on the collection of the ZOFRATACNA tariff, 2010-2016 period. Correlational research that aims to demonstrate whether the behavior of border migration dynamics has an impact on the collection of the ZOFRATACNA tariff in the 2010-2016 period projected towards 2019 . The evolution of the quarterly collection of the special tariff $6 \%$, expressed in thousands of dollars, period 2010-2016 and its projection to 2019 has a decreasing trend. The collection of the special tariff of $6 \%$ is not related to the dynamics of border migrations. The ZOFRATACNA has not planned in its management documents the strategies, plans, activities that are aimed at identifying the "business for its customers users", neither to know the tastes and preferences of the "consumers with purchasing power" that visits the Commercial area of Tacna (street markets). There is a high rotation of the managerial cadres of the organic units. There is a $6 \%$ dropout rate of current user-clients, in the last 3 years. The entry of merchandise from Chile, to the Commercial Zone of Tacna, represents on average $35 \%$ of the total imports, generating a high dependence.
\end{abstract}

Keywords: customer, ZOFRATACNA special tariff ,Tacna Commercial Zone

\title{
INTRODUCCIÓN
}

Desde la creación de la ZOFRATACNA, la unidad estratégica de negocios de la Zona Comercial de Tacna ha sido su principal cimiento para el desarrollo del sistema. Se recauda como arancel especial el $6 \%$. ZOFRATACNA es una de los pocos organismos nacionales que no recibe presupuesto del gobierno central para el desarrollo de sus operaciones.

De acuerdo al enfoque trasnacional, respecto a las migraciones en zona de frontera fronterizas aporta conceptos como el fenómeno de la desterritorialización, y flujos poblacionales que cruzan los linderos de demarcación territorial de acuerdo a criterios de pertenencia nacional (Faist, 2012). Los movimientos transfronterizos que ocurren entre ciudadanos de países limítrofes ponen a prueba conceptos como nación, cultura, desarrollo e identidad. (Migración en Arica y Parinacota, José Tomás Vicuña Undurraga y Tomás Rojas Valenzuela, 2015). Según Podestá (2011:128), el paso terrestre entre Perú y Chile, es el segundo cruce internacional más transitado de Sudamérica. La Región Tacna alberga a la única Zona Franca del Perú que tiene dos unidades estratégicas de negocios i) La Zona Franca que atienda actividades productivas y de servicios y ii) La Zona Comercial de Tacna, que es motivo de análisis en esta investigación por ser el origen de la recaudación del arancel. El área comercial de Tacna corresponde a la geografía del distrito de Tacna y Distrito Alto de la Alianza, donde por Decreto Supremo № 021-2003-MINCETUR. Todos los depósitos Francos de ZOFRATACNA estarán exoneradas del IGV, IPM, ISC, así como de todo impuesto creado o por crearse, incluso de aquellos que requieren de exoneración expresa, pagando únicamente un Arancel Especial del 6\%. 
Las Zonas de Tratamiento Especial surgieron a raíz de la necesidad de desarrollar algunas regiones del país, para promover las exportaciones y generar empleo. Las ZEE son diseñadas como una herramienta de comercio exterior, inversión (nacional y extranjera) y de política de incremento de la oferta exportable regional. Tienen como objetivo superar las barreras que impiden la inversión en una economía más amplia, buscando superar políticas restrictivas, falta de gobernabilidad, inadecuada infraestructura, problemas de acceso a la propiedad, entre otros.

En el Perú existen actualmente 4 zonas en funcionamiento, estas son: Zona Franca de Tacna (ZOFRATACNA), ZED Ilo, ZED Paita y ZED Matarani. Sin embargo, existen 2 zonas que ya han sido creadas a través de norma legal, pero que aún no están operativas, estas son la Zona Económica Especial de Puno (Zeedepuno) y Ceticos Loreto. (Metis Gaia, 2013) desarrolló el diagnóstico de las cuatro ZEE de Perú priorizadas para: (I) ZOFRATACNA, (II) ZED Matarani, (III) ZED Ilo y (IV) ZED Paita. La ZOFRATACNA presenta un nivel de desarrollo en infraestructura relativamente alto, cuenta con mayor desarrollo en materia de seguridad, un mayor desarrollo en términos de tecnología. Presenta estructuras orgánicas relativamente eficientes pero cuenta con un exceso de empleados con falta de uniformización de la normativa. La ZOFRATACNA, presenta desventajas en cuanto a capacidad logística. El no contar con un puerto cercano implica una dificultad para los usuarios que muchas veces puede condicionar su instalación. El 66\% de las empresas instaladas en las ZEE se ubica en la ZOFRATACNA. El 94\% de las importaciones realizadas a través de las ZEE, ingresan por la ZOFRATACNA y el $94 \%$ de las exportaciones realizadas en las ZEE, se efectúan a través de la ZED Paita.

Desde el siglo XIX entre Tacna y Arica hubo un proceso que permitió la complementariedad mucho más allá de lo económico, fue también político y cultural (González, 2006). El vertiginoso crecimiento poblacional se vio influido por la implementación del proyecto minero de Toquepala y la dinamización o la intensificación de las actividades comerciales a partir de la creación de una Zona Franca de Iquique y, posteriormente, la de Tacna. Tacna tuvo que dinamizar su economía, produciendo una demanda de mano de obra. (Dinámicas migratorias en la frontera Perú-Chile, Isabel Berganza Setién, 20111). (Berganza y Cerna, 2011) “La migración es uno de los fenómenos importantes que se encuentra presente en muchas agendas políticas de países a nivel mundial e influye en la globalidad de la vida de las personas, igual sucede en las sociedades de acogida o de destino". Vega Bequer (2014), refiere que más del 60\% de habitantes de Tacna nació en Puno. En contra posición, cerca de 63,360 tacneños migran a otras ciudades del país. (Berganza y Cerna, 2011) refiere que existe una realidad de movilidad humana muy dinámica entre estas dos poblaciones.

\section{OBJETIVOS}

- Conocer el modelo econométrico que se ajusta a la dinámica de las migraciones fronterizas en el periodo 2010-2016 y su proyección al 2019.

- Conocer el modelo econométrico que se ajusta a la recaudación del arancel de ZOFRATACNA periodo 2010-2016 y su proyección al 2019.

\section{METOdOLOGÍA}

Investigación correlacional, que evalúa la relación entre la dinámica de las migraciones fronteriza y la recaudación del arancel de ZOFRATACNA. El diseño de la investigación es no experimental y longitudinal. Recolecta información estadística a nivel regional, nacional e internacional, con incidencia en Tacna (Perú) y Arica (Chile), y a nivel institucional de la Zona Franca y Zona Comercial de Tacna éntrelos años 2010 al 2016, con proyección al 2019. Las unidades de estudio serán los migrantes y la Zona Franca y Zona Comercial de Tacna, así como la Recaudación por el arancel de ZOFRATACNA en el periodo 2010-2016. Se utilizó como técnica el análisis de documentos públicos y privados, así como el análisis del contenido de archivos físicos o electrónicos del periodo 2010-2016. 


\section{RESULTADOS}

Las importaciones que realizan los usuarios de ZOFRATACNA con destino hacia la Zona Comercial de Tacna (mercadillos) tiene una tendencia decreciente muy marcada especialmente a partir del año 2013 que cae en -3\% comparado con el año anterior, y sigue disminuyendo en 2014: - $17 \%, 2015 \%$ : -8\%, 2016\%: -5\% (Tabla 01).

Tabla 01: Importaciones hacia la Zona Comercial de Tacna (\$ CIF)

\begin{tabular}{cr}
\hline Año & Im portaciones \\
\hline 2010 & $126,182.34$ \\
2011 & $138,093.51$ \\
2012 & $139,953.77$ \\
2013 & $135,278.97$ \\
2014 & $112,506.41$ \\
2015 & $103,975.14$ \\
2016 & $98,626.14$ \\
\hline
\end{tabular}

Nota. Fuente: Zo fratacna (2017) O btenido del Procesamiento SP 100130 17 en atención a solicitud de inform ación y pago correspondiente en Tesoreria

Durante el periodo de análisis 2010-2016 la cantidad de empresas usuarias activas -aquellas que realizaron operaciones por ZOFRATACNA- con destino hacia la Zona Comercial (mercadillos) presentó una caída de -16\% a partir del año 2012, según se muestra disminuyó a partir del 2012, pasando de 1933 usuarios activos a 1616 en el año: 2016, lo cual representa 317 clientes menos que realizaron importaciones por el sistema ZOFRATACNA hacia la Zona Comercial. Cabe resaltar que de los 10 tipos de usuarios de ZOFRATACNA, los usuarios de Zona Comercial son los más representativos para el sistema, situación que aún preocupa más (Fig. 01)

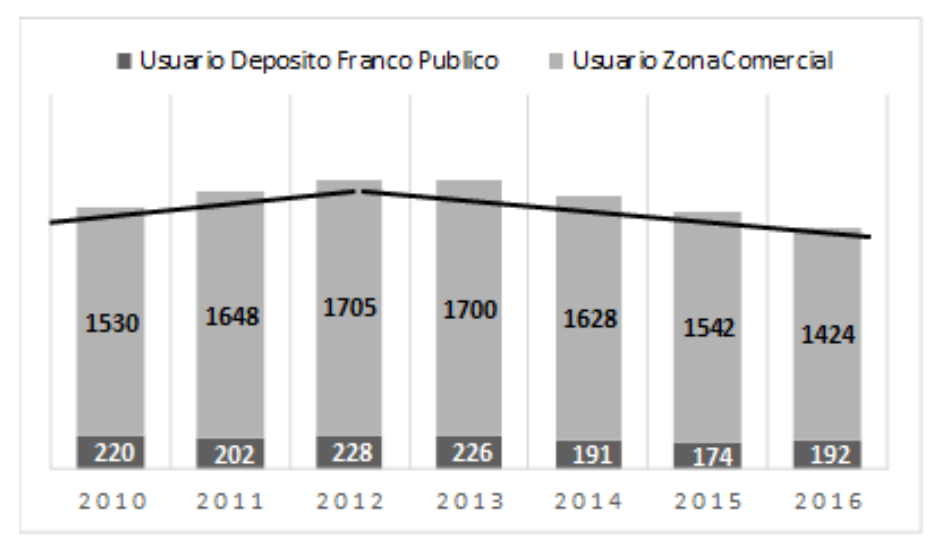

Fig. 01: Evolución de la empresas usuarias de la ZOFRA Tacna

En el acumulado del 2010 al 2016, se observó un descenso en la recaudación del arancel especial del 6\%, analizamos esta caída por año, se registró un descenso a partir del año 2013 ( -3\%), 2014 ( -15\%), 2015 ( -7\%) , 2016 ( -5\%), en promedio del 2013 al 2016 ha caído en -8\%, lo cual representa una gran amenaza para el funcionamiento de la ZOFRATACNA (Fig.02). 


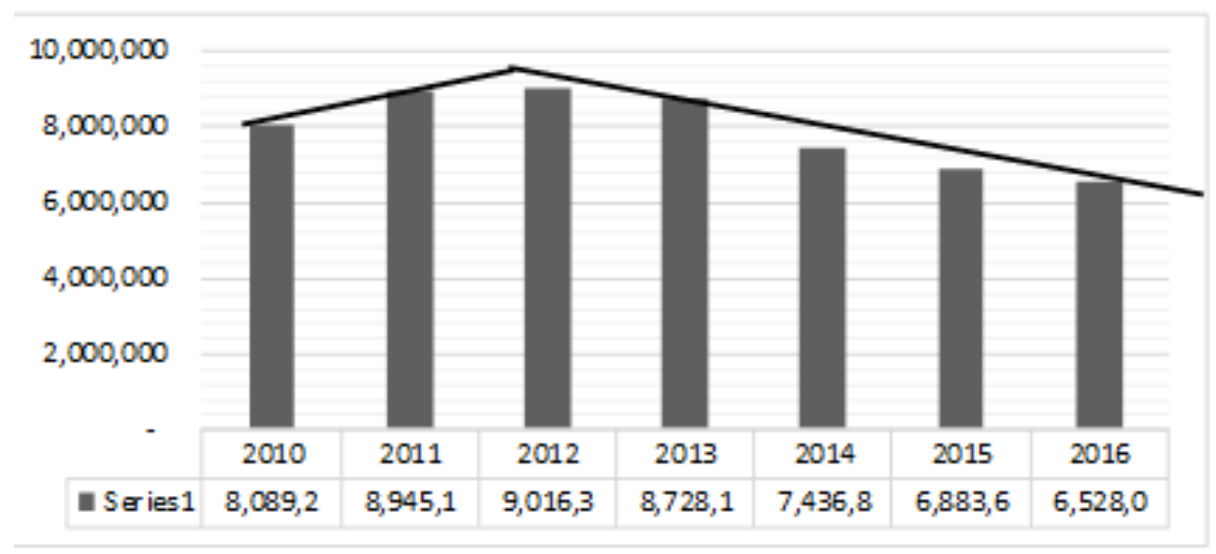

Fig. 02: Evolución de la recaudación del 6\%

De los 10 organismos beneficiarios del Arancel Especial de ZOFRATACNA, no todos se ubican en la Región Tacna, y como consecuencia de la disminución de la recaudación, en la distribución todos los organismos se están viendo perjudicados al recibir menos dinero como consecuencia de los depósitos quincenales que realiza la ZOFRATACNA en cumplimiento del DS N021-2003-MINCETUR. El Proyecto Especial Tacna sería el organismo que desde el inicio del sistema como ZOTAC es el que más dinero ha recibido, beneficiándose con el $40 \%$ del total recaudado por el sistema. Del periodo de análisis del 2010 al 2016 se le ha depositado el equivalente en soles de más de US\$ 21 millones de dólares, en segundo lugar esta ZOFRATACNA que ha recibido el $26 \%$ (más de US\$ 14 millones de dólares) y la ZED ILO, ex Ceticos Ilo con $10 \%$ (más de U\$ 5.5 millones de dólares) que no está ubicado en la región Tacna y que al ser una Zona Económica en Perú, es considerada competencia directa para el sistema ZOFRATACNA. En 4to lugar están los organismos tacneños el Gobierno Regional de Tacna y la Municipalidad Provincial de Tacna, ambos con más de U\$ 3.8 millones de dólares.

Respecto al movimiento migratorio de ingreso de ciudadanos extranjeros por el control de frontera Santa Rosa en Tacna, periodo 2010-2016, expresado en número de personas (Fig. 03), se ha registrado un crecimiento sostenido superior al 10\%, siendo el promedio anual de 1.5 millones de personas.

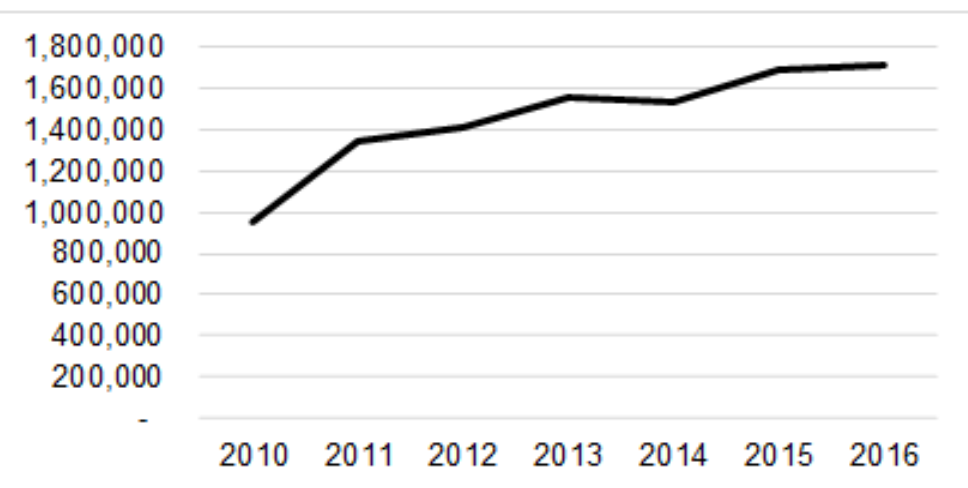

Fig. 03: Movimiento Migratorio 
Según el Instituto Nacional de Estadística e Informática, la población de Perú alcanzó los 31,151,643 millones de habitantes en el año 2015, y la Región Tacna al ser una de las regiones más pequeñas del Perú representa menos del 1.2\% y durante el período de análisis de ésta investigación ha presentado un crecimiento promedio anual de $2 \%$. Asimismo, la población urbana que representa el $91 \%$ se impone sobre la población rural, siguiendo la tendencia nacional. Por otro lado, la gran mayoría de la población vive en la provincia de Tacna y se concentra en primer lugar en el distrito de Tacna, con el (37,7\%); Coronel Gregorio Albarracín Lanchipa, (27,6\%); Alto de la Alianza, (14,2\%); Ciudad Nueva, (13,7\%). Según el Instituto Nacional de Estadísticas de Chile, la población de Chile alcanzó los 18,006,407 millones de habitantes en el año 2015, y la Región de Arica y Parinacota representa menos del 1.3\% y durante el período de análisis de ésta investigación ha presentado un crecimiento promedio anual de $2 \%$, si comparamos con los indicadores anteriores de Perú, observamos que son muy similares.

La evolución de la recaudación trimestral del arancel especial 6\%, expresado en miles de dólares en el periodo 2010-2019 por trimestre es decreciente, según el modelo elegido y la simple observación de los datos proyectados al año 2019 asi como que de acuerdo a los resultados obtenidos del modelo se puede concluir que el movimiento migratorio de ciudadanos extranjeros ingresados por el Control Santa Rosa (210-2016) es creciente (tabla 02).

Tabla 02: Recaudación anual y movimiento migratorio 2010-2016

\begin{tabular}{ccr}
\hline AÑO & \multicolumn{1}{c}{ RECUD } & \multicolumn{1}{c}{ PBMG } \\
\hline 2010 & $8,089,922.00$ & $949,751.00$ \\
2011 & $8,945,158.00$ & $1,338,023.00$ \\
2012 & $9,016,340.00$ & $1,402,987.00$ \\
2013 & $8,728,160.00$ & $1,552,529.00$ \\
2014 & $7,436,811.00$ & $1,530,418.00$ \\
2015 & $6,883,619.00$ & $1,683,800.00$ \\
2016 & $6,528,054.00$ & $1,709,360.00$ \\
\hline
\end{tabular}

Fuente: Archivos físicos y electrónicos 2010-2016 ZOFRATACNA

Correlaciones

\begin{tabular}{|l|l|l|}
\hline & RECUD & PBMG \\
\hline RECUD & & -0.5136 \\
\hline & & $(7)$ \\
\hline & & 0.2384 \\
\hline PBMG & -0.5136 & \\
\hline & $(7)$ & \\
\hline & 0.2384 & \\
\hline
\end{tabular}

No existe correlación significativa al 95\% de confiabilidad. El incremento de la población de migrantes por la frontera de Santa Rosa no influye en la recaudación del arancel en la ZOFRATACNA. 


\section{DISCUSIÓN}

La evolución de la recaudación trimestral del arancel especial 6\%, de ZOFRATACNA, expresado en miles de dólares, proyectada hacia el año 2019 tiene una tendencia decreciente, que se reflejará en menores ingresos para la Administración de ZOFRATACNA, que como hemos analizado durante el periodo 2010-2016, representó en promedio el 77\% del total de sus ingresos que requiere para su funcionamiento, siendo el más representativo los gastos de personal que equivalen al $20 \%$ anual. La entidad tiene una fuerte dependencia al arancel especial $6 \%$ para su continuidad y operatividad. Los 10 organismos nacionales beneficiarios recibirán menos dinero en sus depósitos quincenales, amenaza que se suma a otras variables del entorno externo por las que también recibirán menos depósitos por canon minero y regalías, como consecuencia menos dinero para realizar obras para la región Tacna. La política de integración fronteriza que desarrolla y promueve Perú y Chile, desde sus gobiernos supera los problemas geopolíticos y fomenta la integración regional hacia la libre circulación de bienes, servicios, personas generando un turismo comercial, de salud, gastronómico que contribuye al desarrollo socioeconómico de ambas regiones, y con una proyección hacia el 2019 se concluye que la tendencia del comportamiento de la dinámica de las migraciones fronterizas de ciudadanos extranjeros que ingresan por el puesto de Control fronterizo Santa Rosa será creciente. El visitante del día chileno que arriba a Tacna, realiza un turismo de salud $34 \%$, turismo gastronómico $35 \%$ y $22 \%$ por turismo de compras, siendo el lugar más visitado para las compras la Feria Caplina (42\%), Mercado Central (33\%), Mercadillo 28 de julio (21\%) siendo su capacidad de gasto por día de U\$ 83 dólares en promedio, representando un potencial "consumidor "para los mercadillos de Tacna.

Actualmente la ZOFRATACNA no cuenta con estudios que investiguen la dinámica de las migraciones fronterizas, siendo la Región Tacna un principal punto de ingreso de turistas extranjeros; no se ha puesto en valor el potencial de "consumidor chileno con capacidad de compra", asimismo no existe una oferta comercial diversificada de productos importados en los mercadillos dirigida a éste importante segmento que tiene un salario mínimo vital que duplica el de un consumidor peruano. Y como entidad está perdiendo clientes - usuarios de la Zona Comercial de Tacna, con una tasa sostenida de $-6 \%$ los últimos tres años.

Se propone realizar estudios de inteligencia comercial para identificar el "modelo de negocio" para los usuarios de ZOFRATACNA, conocer y promover las subpartidas que "generan valor" que tienen mayores ventajas y son demandadas por los ciudadanos extranjeros que ingresan por el puesto de Control fronterizo Santa Rosa, de tal manera que se informe, oriente y asesore a los usuarios de ZOFRATACNA que realizan importaciones con destino hacia la Zona Comercial. Para que la recaudación del arancel especial de ZOFRATACNA se relacione y se beneficie con la migración fronteriza. Hay la necesidad urgente de hacer gestiones y remitir al MINCETUR la información necesaria para cambiar la distribución del arancel especial del 6\%. Porque, habiendo transcurrido más de 14 meses de la modificación de la Ley N³0446, "Articulo 9, que modifica la distribución del arancel especial "facultó a ZOFRATACNA a deducir un porcentaje para efectos de solventar los gastos administrativos, operativos y de mantenimiento, y luego recién a partir del saldo realizar la distribución a los diez organismos beneficiarios. Por lo tanto, la entidad es la única responsable que dicha modificación, que los autos benefician, se aplique. Paralelamente, la entidad bien podría trabajar una propuesta de modificación de la Ley que declare la distribución del Arancel Especial del 6\% sólo para entidades de la Región Tacna para el uso "exclusivo" de obras. La ZOFRATACNA debe enfocarse en trabajar las propuestas a remitir al MINCETUR sobre lo que puede ingresar a la Zona Comercial de Tacna, y la franquicia de compra, porque según la modificación de la Ley $N^{\circ}$ 30446, promulgada en junio del 2016 se actualizará cada dos años en base a la propuesta del Comité de Administración. A la fecha han transcurrido más de 14 meses que la responsabilidad de esta gestión en beneficio de la propia entidad les corresponde. 
La recomendación para el Comité de Administración y las unidades orgánicas de ZOFRATACNA sería preguntarse ¿Cuál es el papel del marketing entre el cliente y la ZOFRATACNA? ¿Qué función está cumpliendo el marketing en la dirección de las unidades orgánicas de ZOFRATACNA?. Y luego de dicha reflexión elaborar un Plan Estratégico Institucional que incluya las estrategias para cada unidad de negocio (Zona Franca y Zona Comercial), con una orientación hacia el "cliente-usuario de ZOFRATACNA" como elemento central de la mentalidad de la entidad, dándole valor al marketing estratégico y operativo, plantearse acciones bajo una estrategia "proactiva" elaborando Planes de Marketing, Plan Comercial, Plan de Eventos y otros con la respectiva aprobación oportuna de sus presupuestos para ejecutar las actividades acompañadas de indicadores y planes de control para el logro de los objetivos de cada unidad de negocios.

\section{REFERENCIAS BIBLIOGRÁFICAS}

Berganza y Cerna (2011) Dinámicas migratorias en la frontera Perú-Chile, Arica, Tacna e Iquique. García Jimenez, Jesus (2004) La Comunicación Interna, Madrid, España, Editorial Díaz de Santos S.A. Hill y Jones (2011) Administración estratégica, un enfoque integral, Australia, Cengage

El Instituto Peruano de Economía (2017) Índice de Competitividad Regional - INCORE

Instituto Nacional de Estadísticas de Chile, Dirección Regional de Arica y Parinacota (2015) Enfoque Población.

Instituto Nacional de Estadística e Informática, (2017) Producto Bruto Interno por Departamentos 20072016

Instituto Nacional de Estadística e Informática, (2016) Estadísticas de la Emigración Internacional de Peruanos e Inmigración de Extranjeros, 1990 -2015

Learning Editores S.A. de C. V. México

Jambrino et al. (2008) Manual de Marketing, Madrid, España, ESIC Editorial, 35, 37, 40, 41

Metis Gaia Science and Engineering for Development (2013) Diagnóstico de las Áreas operativas de las Zonas Económicas Especiales del Perú

Municipalidad de Arica (2016) Plan de Desarrollo Comunal Arica 2016-2020

Saínz de Vicuña, José María, (2012) El Plan Estratégico en la Práctica, Madrid, España, ESIC Editorial, 30,195

Saínz de Vicuña, JM, (2012) El Plan de Marketing en la Práctica, Madrid, España, ESIC Editorial, 33, Spencer Milton. H. (2003) Economía Contemporánea, Barcelona España, Editorial Reverte S.A.

Thompson, Strickland y Gamble (2007) Administración estratégica, México, MC Graw Hill Editorial

\section{NORMAS LEGALES}

Decreto Supremo N002-2006-MINCETUR y sus modificatorias; aprueba el Texto Único Ordenado del Reglamento de la Ley N²7688 - Ley de Zona Franca y Zona Comercial de Tacna, Diario Oficial El Peruano, 11 febrero del 2006.

Decreto Supremo N¹53-2007-EF, modificar el D.S. N²02-92-EF, que aprueba la relación de bienes que podrán ser adquiridos al detalle por turistas que visitan la Zona Comercial de Tacna, Diario Oficial el Peruano, 01 de octubre, 2007

Decreto Supremo N019-2009-PCM; aprueba el Reglamento de la Ley N²9014 - Ley de Adscripción de ZOFRATACNA al Gobierno Regional de Tacna, Diario Oficial El Peruano, 28 marzo del 2009.

Decreto Supremo № 006-2012-MINCETUR; modifican el Texto Único Ordenado del Reglamento de la Ley № 27688 Ley de Zona Franca y Zona Comercial de Tacna Ordenanza Regional N004-2008CR/GOB. REG.TACNA; aprueba el Reglamento de Organización y Funciones del Comité de Administración de ZOFRATACNA, Diario Oficial El Peruano, 13 marzo del 2012. 
Decreto Supremo N¹78-2012-EF, Aprueban ampliación y adecuación con el Arancel de Aduanas 2012 de la lista de mercancías que pueden ingresar a la Zona Comercial de Tacna, Diario Oficial El Peruano, Lima, Perú, 18 de setiembre del 2012.

Decreto Supremo N063-2013-EF, modificar el D.S. N²02-92-EF, que aprueba la relación de bienes que podrán ser adquiridos al detalle por turistas que visitan la Zona Comercial de Tacna, Diario Oficial el Peruano, 28 de marzo del 2013.

Decreto Supremo N²53-2013-EF, Aprueban ampliación de la lista de mercancías que pueden ingresar a la Zona Comercial de Tacna, Diario Oficial El Peruano, Lima, Perú, 12 de octubre del 2013.

Ley $N^{\circ} 27688$ y sus modificatorias; Ley de Zona Franca y Zona Comercial de Tacna, Diario Oficial El Peruano, 28 marzo del 2002.

Ley № 28528, Ley que regula el traslado de mercancías con destino a la ZOFRATACNA, los CETICOS y terceros países, 25 de mayo del 2005.

Ley № 28599, Ley que modifica la Ley de Zona Franca y Zona Comercial de Tacna -Ley № 27688 y modificatorias, 16 de agosto del 2005.

Ley № 29739; Ley de promoción de inversión en la Zona Franca y la Zona Comercial de Tacna y que modifica la Ley № 27688 Ley de Zona Franca y Zona Comercial de Tacna y Normas Complementarias, Diario Oficial El Peruano, 6 julio del 2011

Ley $N^{\circ} 29778$, Ley Marco para el desarrollo e integración fronteriza aprobado por Decreto Supremo № 017-2013-RE 13, Diario Oficial El Peruano, Lima, Perú, 27 de julio de 2011.

Ley $\mathrm{N}^{\circ} 30446$, Ley que establece el marco legal complementario para las Zonas Especiales de Desarrollo , la Zona Franca y la Zona Comercial de Tacna, Diario Oficial El Peruano, Lima, Perú, 3 junio del 2016.

Ley № 29014; Ley que adscribe ZOFRATACNA al Gobierno Regional de Tacna, Diario Oficial El Peruano, 12 mayo del 2017.

Resolución Ejecutiva Regional N¹66-2015-P.R./ GOB.REG. TACNA; aprueba el Plan Estratégico Institucional del Comité de Administración de ZOFRATACNA 2015-2017, 2 de febrero del 2015.

Resolución Ministerial №122-2004-MINCETUR/ DM; aprueba el Reglamento Interno del Comité de Administración de la Zona Franca y Zona Comercial de Tacna -ZOFRATACNA, 2 de abril del 2004. 